\section{Swedish Physics through the Looking Glass}

\section{Lars Bergstrom of the Department of Physics, Stockholm University, summarises the report $^{\star}$ of a recent international evaluation of physics in Sweden.}

There has been a debate in Sweden the last two years, partly in newspapers and other media, concerning the quality and profile of Swedish physics. With the Swedish public being more and more engaged in the question of Sweden's eventual participation in the European Community, many aspects of Swedish society are being continuously scrutinised and compared with their counterparts in other European countries. So also the universities and, in particular, their physics departments. Sweden has in fact been a member for some time of several of the EC programmes for science and higher education, and a non-negligible fraction of the government's research funds are already being channelled through these programmes. Since many see this as the beginning of a general trend where Swedish researchers must obtain their grants in competition with other European scientists, it was considered important to evaluate the competitiveness of Swedish science, as seen from the outside.

To this end, the Swedish Natural Science Research Council (NFR) decided, in the spring of 1991 , to call together a committee consisting of prominent international physicists with the task of performing a thorough evaluation of Swedish physics. The members of the committee were Alexander Bradshaw (Berlin), Nicola Cabibbo (Rome), Alexander Dalgarno (Harvard), Paul Kienle (Darmstadt), Norman F. Ramsey (Harvard) and John Wilkins (Ohio), with Lennart Eberson (Lund), a Swedish chemist, as Chairman and Finn Karlsson (NFR) as the Secretary. They were aided by a reference group consisting of Swedish scientists (including the present author) from the various sub-fields of physics. A detailed questionnaire covering all essential aspects of financing, group structure, international contacts, publishing, etc. that was sent to all research groups resulted in an extensive collection of data on Swedish physics. In addition, the committee interviewed several key people (faculty deans, Directors of large installations, etc.).

The committee's report*, which was made public last spring, comprises a general review of Swedish physics, descriptions of the large research facilities, a thorough analysis of financial and statistical indicators, discussions of the balance between fields and the "sociology" of Swedish physics, and, finally, the committee's conclusions. The novelty of the evaluation compared to numerous others performed by the NFR is that it spanned the whole of physics (including astronomy and space physics). Neither individual projects nor specific research groups or departments were evaluated.

The committee chose not to take a stand on the somewhat controversial question of an imbalance between sub-fields claimed by some. In particular, it has been argued that modern branches of physics such as particle physics and some condensed matter physics are under represented, with, e.g., nuclear and atomic physics being over represented. The committee's view was that there are no absolute standards: research in different countries develops differently due to various internal and external factors. However, it noted the need to constantly compare the Swedish profile with those of other countries, arguing at the same time that such comparisons must not be decisive for the future direction of Swedish physics.

The overall quality of Swedish physics is high from an international perspective. A bibliometric study containing, e.g., a citation analysis, as supporting evidence, established that Swedish physics is internationally very competitive even though the total support for the basic branches of natural science seems to be lower in Sweden than in comparable countries. Expanding research fields should be financed by an increase of the physics budget rather than by a redistribution of existing resources.

International, and in particular European, collaborations are highlighted. A considerable fraction of total research budget for physics meets annual contributions to large facilities such CERN, ESO, JET, ESRF, etc. The report emphasises the importance for a small country like Sweden to take part in these endeavours, to obtain knowledge of state-of-the art science and technology and to take back this knowledge to home institutions. The report wonders if the home-based experimental particle physics community is experience gained at CERN. One recommendation is, indeed, that experimental particle physics in Sweden should grow.

A central board should be created to organize and finance the country's several fairly large accelerator and storage ring facilities. This would eliminate the risk of underfinancing many simultaneous projects, a tendency which has been seen in Sweden, incompetitive facilities. Efforts should be made large enough to be able to fully exploit the stead of putting more resources into the most to attract more foreign scientists to make extended visits, and especially to collaborate with Swedish groups at national facilities.

One area, namely the academic career structure, was singled out for quite explicit criticism. The report speaks of "an extreme pyramidal situation which denies permanent positions too long to those who deserve them and at the same time overloads the permanent persons with so many administrative responsibilities that the teaching is neglected by loading it onto a few." Surprisingly, positions of the "tenure track" type do not exist in the Swedish system and the expression "the missing associate professor" was coined.

A completely new career system is sketched based on components that are wellknown in many other countries, including a full-fledged post-doc system and a system of tenure-track associate professors, with decision concerning tenure taking place before the applicant is 35 years old and thus still sought after outside the university system.

Needing a remedy is the problem of a very low mobility of people between universities, both within Sweden as well as between Sweden and the rest of Europe. There is a strong tendency for "inbreeding" meaning, for example, that it is not uncommon for a person to remain at the same university from undergraduate studies until retirement as a lecturer or professor. Since this is difficult to remedy in isolation in a small country like Sweden with its relatively few universities, one may hope that the various European mobility schemes will be effective to this end.

In summary, Swedish physics is quite strong by international comparisons. Very good, and in some areas excellent, research has been established with relatively limited resources. If authorities implement the improvements suggested by the evaluation committee, Swedish physicists may look to the future with confidence. The "experiment" of an evaluation of physics with a broad scope is generally regarded to have been successful so the NFR is now making similar evaluations of other branches of science.

* International Evaluation of Swedish Research in Physics. From: NFR, Box 6711, S-113 85 Stockholm [Fax: +46 (8) 61007 40].

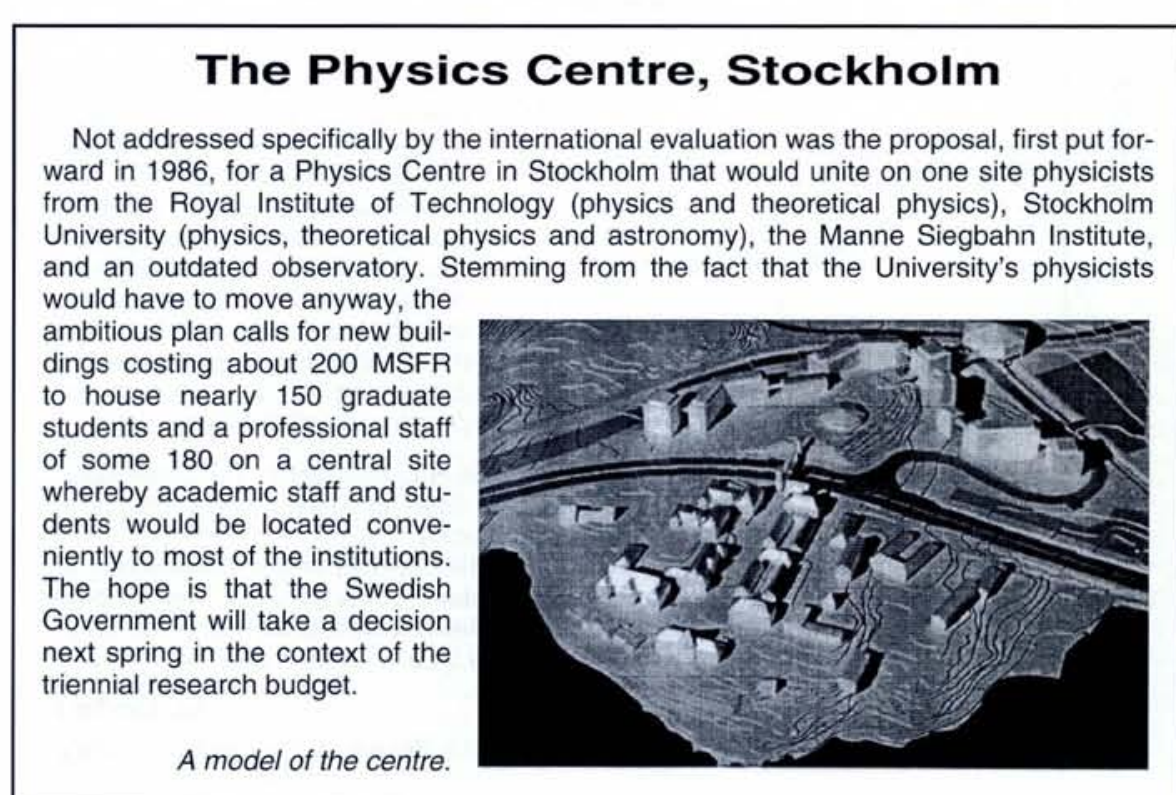

